\title{
Comparison of radius of anterior lens surface curvature measurements in vivo using the anterior segment optical coherence tomography and Scheimpflug imaging
}

\author{
Zhenzhen Liu", Xiaoting Ruan", Wei Wang", Juzi Liu, Yijing Meng, Xiaoxun Gu, Jun Fu, Lixia Luo, \\ Yizhi Liu
}

State Key Laboratory of Ophthalmology, Zhongshan Ophthalmic Center, Sun Yat-sen University, Guangzhou 510060, China

Contributions: (I) Conception and design: Z Liu, L Luo; (II) Administrative support: Y Liu; (III) Provision of study materials or patients: X Ruan, W Wang; (IV) Collection and assembly of data: J Liu, Y Meng; (V) Data analysis and interpretation: X Gu, J Fu; (VI) Manuscript writing: All authors; (VII) Final approval of manuscript: All authors.

\#These authors contributed equally to this work.

Correspondence to: Lixia Luo, MD, PhD. State Key Laboratory of Ophthalmology, Zhongshan Ophthalmic Center, Sun Yat-sen University, Guangzhou 510060, China. Email: luolixia@gzzoc.com

Background: To assess the radius of anterior lens surface curvature (RAL) measurements with anterior segment optical coherence tomography (AS-OCT) in comparison with Scheimpflug imaging.

Methods: This prospective, cross-sectional study was carried out at Zhongshan Ophthalmic Center, Guangzhou, China. We enrolled 59 eyes, including 30 eyes from 30 cataractous volunteers (59 to 87 years) and 29 eyes from 29 young participants (19 to 49 years). After mydriasis, the RAL was measured automatically by the built-in software in the AS-OCT (CASIA 2). The Scheimpflug images were measured with the build-in caliper tool of the Scheimpflug camera (Pentacam), and RAL were further calculated with the principle of best-fitted circle. Intraobserver and interobserver reproducibility of RAL measurement using Scheimpflug camera were evaluated with limit of agreement (LoA) and intraclass correlation coefficient (ICC). Consistency between RAL measurement of Scheimpflug camera and AS-OCT were assessed with LoA, correlation analysis and linear regression.

Results: For all subjects, intraobserver (LoA: -0.25 to $0.23 \mathrm{~mm}$, ICC: 0.996 ) and interobserver reproducibility (LoA: -0.85 to $0.92 \mathrm{~mm}$, ICC: 0.947 ) of RAL were good using Scheimpflug imaging. Both AS-OCT and Scheimpflug imaging found that the age-related cataract participants had smaller RAL ( $\mathrm{P}=0.010, \mathrm{P}=0.001$ respectively). LoA of RAL measurement between AS-OCT and Scheimpflug imaging was -3.83 to $-0.79 \mathrm{~mm}$, and the Pearson correlation efficient was $0.909(\mathrm{P}<0.001)$. The RAL values measured by AS-OCT were significantly greater than that by Scheimpflug camera with a mean difference of $2.31 \mathrm{~mm}$ for all participants $(\mathrm{P}<0.001)$. The RAL measurement could be converted using the equation: $\mathrm{Y}_{\mathrm{CASIA} 2}=1.155 \times$ $\mathrm{X}_{\text {Pentacam }}+1.060$.

Conclusions: Both Scheimpflug camera system with internal caliper tool and the AS-OCT are fast and non-contact tools that could measure RAL successfully. The two measurement results are highly correlated and interchangeable through linear regression equation.

Keywords: Crystalline lens; radius of anterior lens surface curvature (RAL); Scheimpflug Camera; anterior segment optical coherence tomography (AS-OCT)

Submitted Oct 20, 2019. Accepted for publication Jan 14, 2020.

doi: 10.21037/atm.2020.01.100

View this article at: http://dx.doi.org/10.21037/atm.2020.01.100 


\section{Introduction}

The crystalline lens is the most dynamic optical element of the human eyes. The lens changes its surface curvature during accommodation so the eyes can focus on objects at different distances (1). Previous studies have shown that in the process of accommodation, the focus is mainly adjusted by changing the radius of anterior lens surface curvature (RAL), while the radius of posterior lens surface curvature is only slightly or not changed (2). Therefore the accurate measurement of RAL is of paramount importance for biometric studies of accommodation and relative ocular disorders, such as myopia and presbyopia (3-5).

B-scan ultrasonography, high resolution nuclear magnetic resonance imaging (HRMRI), anterior segment optical coherence tomography (AS-OCT) and Scheimpflug photography are the techniques available for the imaging of the crystalline lens in vivo (6-9). However, due to low resolution, difficulties in correcting distortion of different optical interfaces, long scanning time, inconvenient examination setting, and lack of interchangeable standards between instruments, it is difficult to obtain large scale data of RAL measurement in vivo. Therefore, the RAL reference value of the human eyes for different age groups remain unknown, and there is no gold standard for RAL measurement of the human eyes.

Among the above-mentioned techniques, Scheimpflug photography and AS-OCT are potentially appropriate to collect census data of RAL measurements in vivo. Both methods are noncontact and fast procedures. The Scheimpflug instruments are more widely used for refractive evaluation than the AS-OCTs. The commercially available build-in manual caliper of some Scheimpflug instruments allows observer to measure the exact distance in distorted image (10), and using secondary calculation to obtain RAL value may be feasible. High reproducibility of the new generation AS-OCT in measuring lens parameters including RAL has been reported recently $(11,12)$. Additionally, the second generation AS-OCT, CASIA 2 , is the first instrument able to automatically measure and calculate RAL value. Several studies reported results comparing Scheimpflug photography and AS-OCT in biometry for ocular anterior segment (13-16). However, no studies have compared RAL measurement between Scheimpflug photography and AS-OCT. Consistency and transformation of the results from two measurement methods remain to be elucidated.
In this study, we aimed to validate the feasibility of RAL measurement using secondary calculation of Scheimpflug images, and establish a transformation equation of RAL measurement from Scheimpflug camera and AS-OCT. The intraobserver and interobserver reproducibility of Scheimpflug imaging was assessed in all subjects. We then compared the consistency and correlation between RAL measurements obtained with Scheimpflug images and AS-OCT and evaluate whether both methods could detect the age-related changes in RAL.

\section{Methods}

\section{Subjects and settings}

This study was conducted at the Zhongshan Ophthalmic Center, Sun Yat-sen University, Guangzhou, China. Volunteers were consecutively recruited from the Outpatient Department during August 2019 to September 2019. Subjects with any evidence of the following conditions were excluded: (I) ocular diseases affecting anterior structures such as glaucoma, uveitis, lens subluxation and ocular trauma; (II) a history of intraocular surgery; (III) corneal abnormalities that would affect imaging such as leucoma, keratoconus, or corneal scar; (IV) poor fixation leading to low image quality or inability to cooperate with examinations. The study was approved by the ethics committee of Zhongshan Ophthalmic Center and was performed in accordance with the tenets of the Declaration of Helsinki. Written informed consent was obtained from all participants.

\section{Anterior segment scanning}

Anterior segment scanning was performed with an CASIA2 (Tomey Corporation, Nagoya, Japan) as well as a Scheimpflug camera, Pentacam (Oculus, Wetzlar, Germany). These two measurements were performed independently by two experienced operators (J Liu and Y Meng) in random sequence. The operators were masked to one another's results. The measurements were performed with pupil dilation using topical $1 \%$ tropicamide in the same dark room. During CASIA2 imaging, the subjects were asked to fixate on the target. CASIA2 measurements of RAL were aligned along the visual axis and performed using the 16-scan lens biometry mode. The measurements of RAL were automatically generated by the "Lens Analysis" module in "Lens Biometry" mode. After CASIA2 


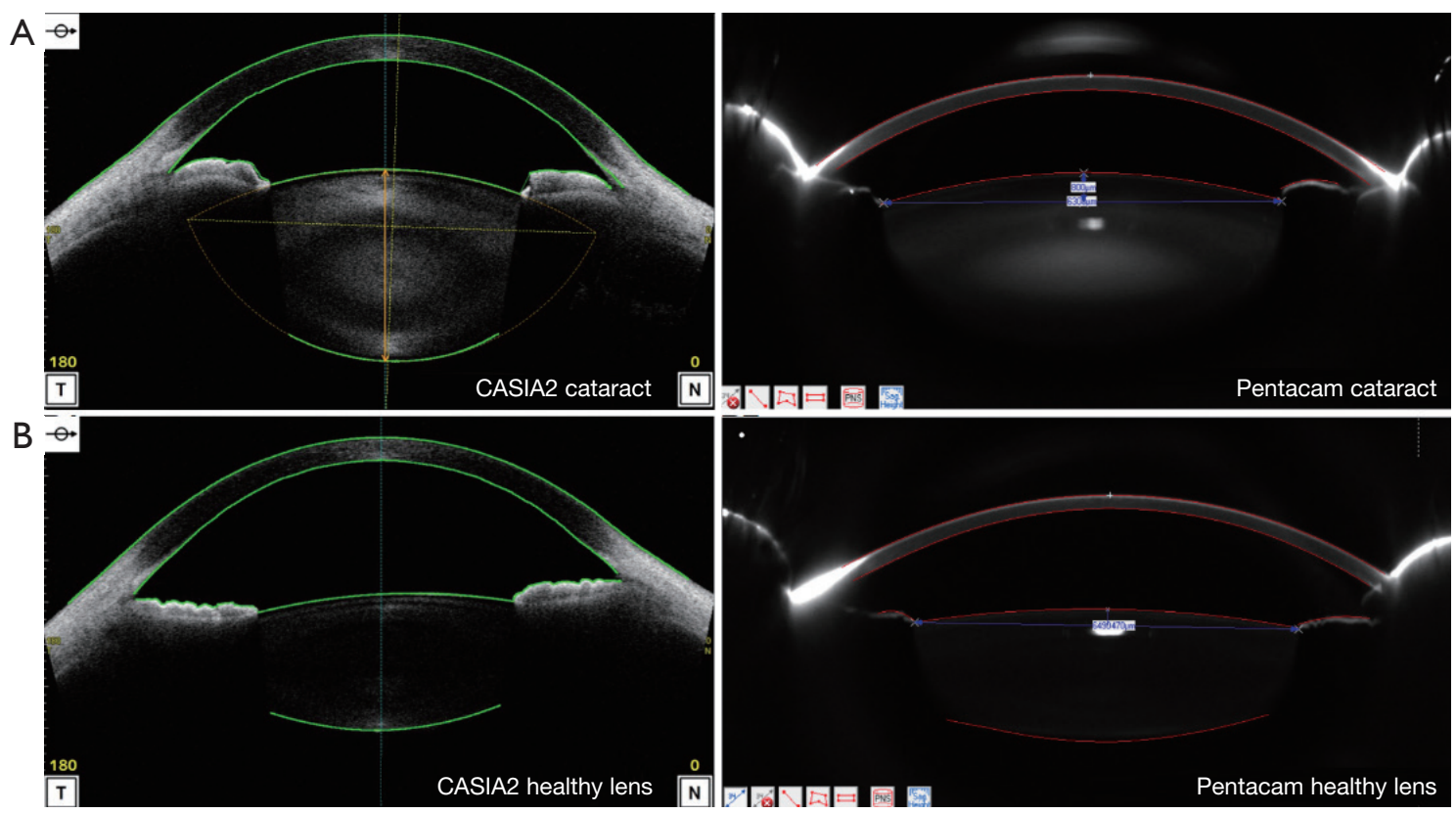

Figure 1 Lens anterior curvature radius measurement by AS-OCT and rotating Scheimpflug Camera. (A) The same cataractous subject scanned with AS-OCT and Pentacam; (B) the same young subject scanned with AS-OCT and Pentacam.

scanning, a measurement with Pentacam was performed using the 25 -scan acquisition mode in the same dark room. Figure 1 shows the representative images obtained by two instruments for the same subject. The acquisition with the qualified centrality was selected for further measurement and calculation of RAL.

\section{RAL calculation using Scheimpflug images}

Two ophthalmologists (X Gu, J Fu) independently measured the length of $\mathrm{H}$ and $\mathrm{L}$ in the images by using the Pentacam's build-in manual caliper tool (Figure 2A). Each selected scan was repeatedly measured by one ophthalmologist for two times. $\mathrm{L}$ was defined as half of the length between two junctions of the lens anterior capsule and the pupil margin after mydriasis. $\mathrm{H}$ was defined as the length of the vertical bisector of the connection between two junctions of the lens anterior capsule and the pupil margin intersecting with the lens anterior capsule (Figure 2B). Three horizontal sectional scans of the same eye were chosen for image measurement, and the mean $\mathrm{L}$ and $\mathrm{H}$ were used to calculate the radius of the best fitting circle. According to the Pythagorean theorem, the RAL, defined as the radius of the best-fitted circle (referred to as $\mathrm{R}$ in the image) was calculated as $\left(\mathrm{L}^{2}+\mathrm{H}^{2}\right) / 2 \mathrm{H}$. The lens anterior surface with the diameter of $5-7 \mathrm{~mm}$ in the pupil area was included.

\section{Data analysis}

The data of the right eyes were used for analysis. All continuous variables were described as mean \pm standard deviation. The reproducibility assessment was based on the definitions adopted by the British Standards Institution (17). Intraclass correlation coefficients (ICCs) and the limit of agreement (LoA) were used to assess intraobserver and interobserver reproducibility of Pentacam. The average RAL calculated from three scans of one eye was used to compared with the $3 \mathrm{D}$ result of RAL measurement from CASIA2. The paired t-test, the LoA, Pearson correlation analysis and Bland-Altman plot were used for comparing reproducibility, as well as the consistency between Pentacam and CASIA2. The linear regression was used to construct equation for RAL measurement conversion between Pentacam and CASIA2. All statistical analyses were performed using SPSS statistical software (SPSS Statistics version 22.0; IBM Corp., Armonk, NY, USA). Statistical significance was defined as $\mathrm{P}<0.05$.

\section{Results}

In this study, we enrolled 59 volunteers (59 eyes). The pipeline of the study is shown in Figure 3. Among them, there were 30 patients with age-related cataract (30 eyes), 


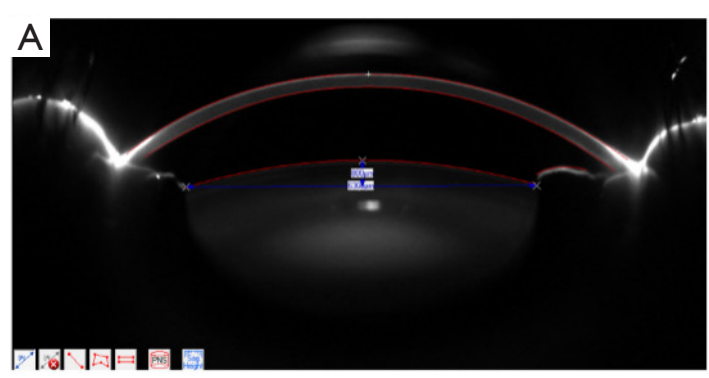

B

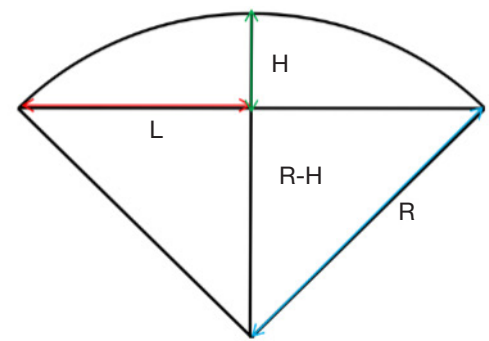

Figure 2 The process of measuring and calculating the anterior surface curvature of the lens by Scheimpflug Camera. (A) The Pentacam's build-in manual caliper tool was used to measure the exact length of $\mathrm{H}$ and $\mathrm{L}$ in the Scheimpflug images. (B) $\mathrm{L}$ was defined as half of the distance between two junctions of the lens anterior capsule and the pupil margin after mydriasis. $\mathrm{H}$ was defined as the distance between the intersection of the vertical bisector of the connection between two junctions of the lens anterior capsule and the pupil margin and the intersection of the lens anterior capsule. $\mathrm{R}$ was defined as the radius of the best-fitted circle.

the mean age was $69.97 \pm 8.83$ years; 29 young subjects (29 eyes) were included with age of $29.22 \pm 9.75$ years. CASIA2 and Pentacam measurements were measurable in all subjects. Baseline characteristics of the subjects were summarized in Table 1.

Table 2 shows the analysis of intraobserver reproducibility and interobserver consistency of RAL measurements using Scheimpflug images by two examiners in elderly and young subjects. The overall intraobserver LoA ( -0.25 to 0.23$)$ was much smaller than that of interobserver LoA ( -0.85 to 0.92). The same trend was confirmed by the ICC (overall intraobserver: 0.996 vs. overall interobserver: 0.947). The interobserver LoA of RAL measurement in the elders $(-0.50$ to 0.48 ) was smaller than that of young subjects ( -1.03 to 1.16$)$.

The mean difference between CASIA2 and Pentacam was $2.24 \mathrm{~mm}$ (paired $t$-test, $\mathrm{P}<0.001$ ) in the elderly subjects, and $2.38 \mathrm{~mm}$ (paired $t$-test, $\mathrm{P}<0.001$ ) in young subjects (Table 3). CASIA2 gave greater measures than Scheimpflug photography, and these differences tended to be consistent across the measurement ranges (Figure 4). In elderly people, the Pearson correlation coefficient (r) between AS-OCT and Scheimpflug photography was $0.714(\mathrm{P}<0.001)$. In young subjects, the Pearson correlation coefficient (r) between AS-OCT and Scheimpflug photography was $0.950(\mathrm{P}<0.001)$. Two measurements correlated highly with each other $(r=0.909, \mathrm{P}<0.001)$. The linear regression equations for measurement conversion were as following:

(I) Age-related cataractous eyes: $\mathrm{Y}_{\mathrm{CASIA} 2}=0.9179 \times$ $\mathrm{X}_{\text {Pentacam }}+2.841$;

(II) Healthy young eyes: $\mathrm{Y}_{\text {CASIA 2 }}=1.285 \times \mathrm{X}_{\text {Pentacam }}-$ 0.1322 ;
(III) All: $\mathrm{Y}_{\text {CASIA 2 }}=1.155 \times \mathrm{X}_{\text {Pentacam }}+1.060$.

$\mathrm{Y}$ represented RAL measurement using AS-OCT (CASIA 2), X represented RAL measurement using Scheimpflug imaging (Pentacam).

\section{Discussion}

The RAL plays an important role in deciding and monitoring the refractive power of the lens. However, there is no gold standard for RAL measurement of the human eyes and the range of RAL remains controversial among different studies. We used two instruments designed based on different optical principles and obtained RAL value similar to the range reported in the literature. The average RAL value of all valid data is $10.38 \pm 1.78 \mathrm{~mm}$ for AS-OCT and $8.12 \pm 1.40 \mathrm{~mm}$ for Scheimpflug imaging in our study. Previous studies have reported measurement of RAL in cadaveric eyes or in vivo using different techniques. Rosen et al. measured RAL of 37 human lenses in vitro by using the technique of shadow photogrammetry reported the RAL of the 63-year-old human was $8 \mathrm{~mm}$ (18). Rosales et al. analyzed 46 eyes in vivo (mean age: $30 \pm 9$ years) with Scheimpflug imaging and found a mean RAL of $11.1 \pm 1.1 \mathrm{~mm}$ and $10.8 \pm 1.1 \mathrm{~mm}$ with Purkinje imaging system (19). Koretz et al. used 100 subjects examined with in vivo Scheimpflug photography and found that subjects aging from 18 to 65 years had a central RAL larger than $8.5 \mathrm{~mm}$ at $0 \mathrm{D}$ accommodation (20). Using AS-OCT, Yuan et al. reported a mean RAL of $12.33 \pm 1.14 \mathrm{~mm}$ at nonaccommodative status in 35 young adults (3). Richdale et al. used 26 emmetropic subjects aging from 30 to 50 years and reported a mean RAL value $10.75 \pm 1.27 \mathrm{~mm}$ under 


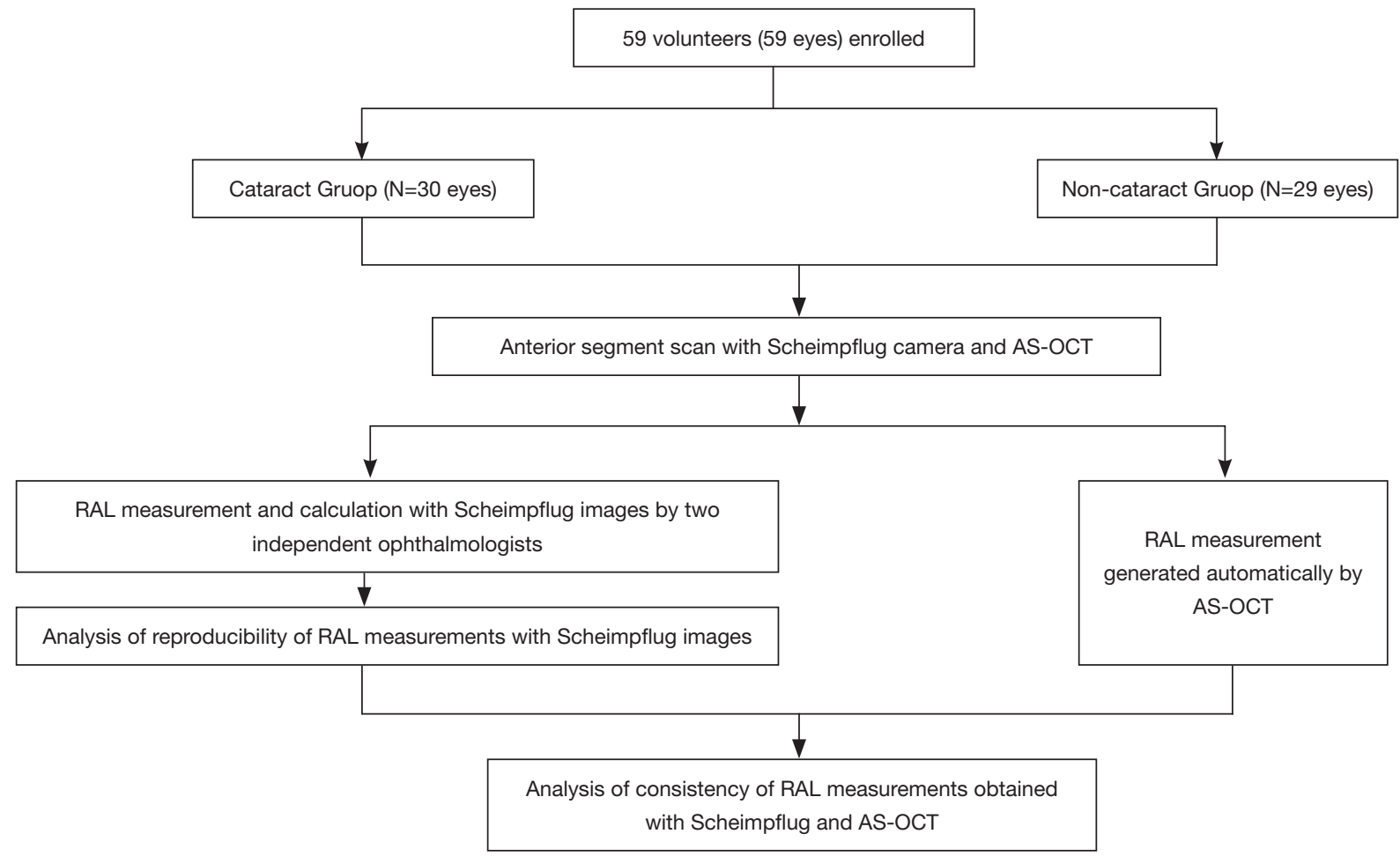

Figure 3 Flowchart of recruitment, measurement and calculation. RAL, radius of anterior lens surface curvature; AS-OCT, anterior segment optical coherence tomography

Table 1 Baseline characteristics of the participants

\begin{tabular}{lcc}
\hline Characteristics & Cataract $(n=30$ persons, 30 eyes $)$ & Healthy lens $(\mathrm{n}=29$ persons, 29 eyes) \\
\hline Age (mean \pm SD, years) & $69.97 \pm 8.83$ & $29.22 \pm 9.75$ \\
Gender, $n(\%)$ & & $2(6.90)$ \\
Male & $8(26.67)$ & $27(93.10)$ \\
Female & $22(73.33)$ & \\
\hline
\end{tabular}

$\mathrm{SD}$, standard deviation.

Table 2 Intra- and interobserver reproducibility of RAL measurements by Pentacam

\begin{tabular}{lccccc}
\hline \multirow{2}{*}{ Group } & \multicolumn{2}{c}{ Intraobserver } & & \multicolumn{2}{c}{ Interobserver } \\
\cline { 2 - 5 } & LoA $(\mathrm{mm})$ & ICC & LoA (mm) & ICC \\
\hline Cataract & -0.25 to 0.20 & 0.986 & -0.50 to 0.48 & 0.941 \\
Non-cataract & -0.26 to 0.26 & 0.995 & -1.03 to 1.16 & 0.914 \\
All & -0.25 to 0.23 & 0.996 & -0.85 to 0.92 & 0.947 \\
\hline
\end{tabular}

RAL, radius of anterior lens surface curvature; LoA, the limit of agreement; ICC, the intraclass correlation coefficient. 
Table 3 Comparison of RAL measured by CASIA 2 and Pentacam

\begin{tabular}{|c|c|c|c|c|c|c|}
\hline Group & $\begin{array}{c}\text { CASIA } 2 \\
\text { (mean } \pm \text { SD), mm }\end{array}$ & $\begin{array}{c}\text { Pentacam } \\
\text { (mean } \pm \text { SD), } \mathrm{mm}\end{array}$ & $\begin{array}{l}\text { Mean difference } \\
(95 \% \mathrm{Cl}), \mathrm{mm}\end{array}$ & LoA, mm & $r$ & $\mathrm{P}$ \\
\hline Cataract & $9.59 \pm 1.17$ & $7.35 \pm 0.91$ & 2.24 (1.93 to 2.54$)$ & -3.85 to -0.63 & 0.714 & $<0.001$ \\
\hline Non-cataract & $11.22 \pm 1.94$ & $8.83 \pm 1.43$ & 2.38 (2.11 to 2.66$)$ & -3.81 to -0.96 & 0.950 & $<0.001$ \\
\hline$P$ & $<0.001$ & $<0.001$ & & & & \\
\hline
\end{tabular}

RAL, radius of anterior lens surface curvature; LoA, the limit of agreement; ICC, the intraclass correlation coefficient.

A

Cataract

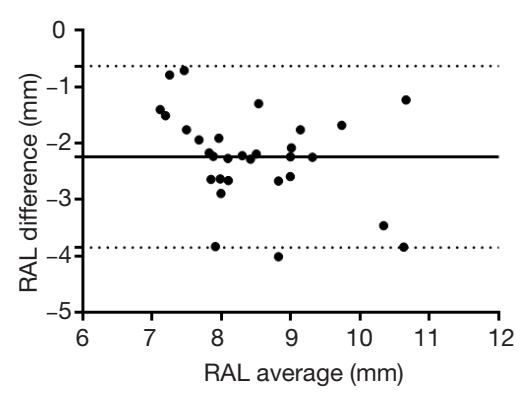

B

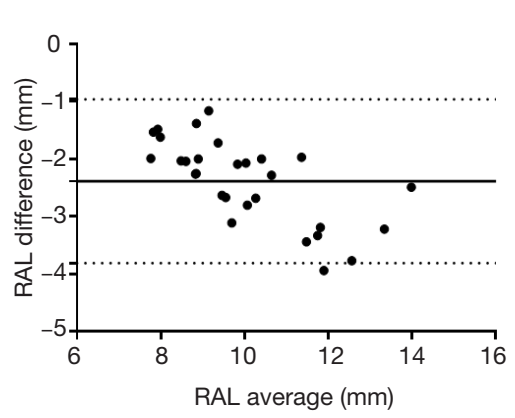

C

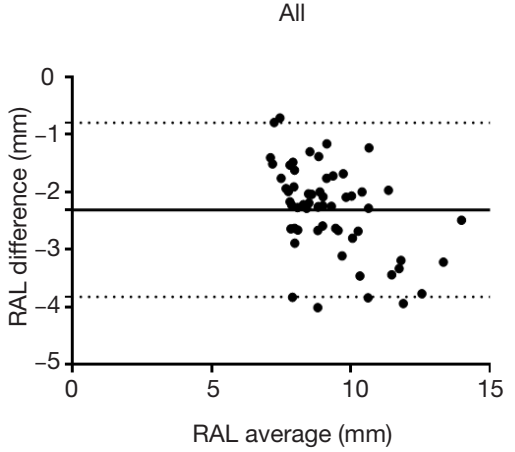

Figure 4 Bland-Altman plots of lens anterior curvature radius by AS-OCT and Scheimpflug Camera. RAL, radius of anterior lens surface curvature.

cycloplegia with phakometry (21). Ramasubramanian et al. analyzed 51 young and mid-age subjects with UBM, and reported a mean RAL of approximate $11 \mathrm{~mm}(22)$. Hermans et al. examined five subjects aging from 18 to 35 years and found a RAL of $11.45 \pm 1.7 \mathrm{~mm}$ (MRI) and $12.15 \pm 0.6 \mathrm{~mm}$ (Scheimpflug imaging) respectively (23). In the abovementioned studies, the RAL was obtained through custombuild instrument, secondary measurement, calibration, and calculation. The instruments and setting used by each research group varied, so did the measurement and calibration algorithms, which makes it difficult to directly compare these measurement results. We can conclude that there is a disparity in RAL measurement resulted from the diversity of instruments and measuring methods.

The built-in caliper tool of Pentacam automatically corrects the distortion of different areas and depths of the image and solves the problem of standardization and uniformity of Scheimpflug image measurement to a large extent. Studies using Pentacam built-in software have suggested a high reproducibility of the secondary measurements for anterior biometrics such as cornea thickness and anterior chamber depth $(24,25)$. Our data show that the interobserver error (LoA: -0.85 to 0.92 ) was greater than that of intraobserver (LoA: -0.25 to 0.23 ). This indicates that the main source of the error originated from the difference between two observers when measuring the same Scheimpflug section with the built-in caliper tool. The stability of the measurement results was improved by measuring one section twice and taking the mean value, and the intraobserver ICC could reach 0.996. Therefore, this error did not result in inconsistency of the two measurement results from the same measurer, because the interobserver ICC reached 0.947. Moreover, we measured the RAL of age-related cataractous subjects and healthy young subjects with AS-OCT and Scheimpflug camera, both instruments were able to detect the decrease of RAL with aging (both $\mathrm{P}<0.001)$. The measurements from two instruments were highly correlated $(\mathrm{r}=0.909, \mathrm{P}<0.001)$, indicating the trend between the two methods is consistent. The CASIA2 is a new generation SS-OCT, several studies have proved the reproducibility and agreement in measuring the biological parameters of the anterior segment and the lens. Chansangpetch et al. evaluated the agreement of anterior segment parameters between CASIA2 and Visante OCT 
and their result showed good agreement in most angle parameters (26). Shoji et al. showed good reproducibility of in vivo crystalline lens measurements in CASIA2 (27). However, we found that the CASIA2 tended to give greater RAL values compared to that obtained from Pentacam image (with a difference of $2.24 \mathrm{~mm}$ in the elderly, $2.38 \mathrm{~mm}$ in young subjects, and $2.31 \mathrm{~mm}$ for all subjects). This constant difference may mainly attribute to the following two reasons. Firstly, these two instruments are designed based on different optical principles. Pentacam uses a blue slit light with a wavelength of $475 \mathrm{~nm}$ for illumination and takes a series of Scheimpflug cross-sectional images of the anterior segment with 360-degree uniform rotation (28). CASIA2 quantifies the distance based on a linear scan, using infrared light and the principles of low-coherence interferometry. Previous study showed that the disparities between AS-OCT and Scheimpflug photography vary in ocular tissues: AS-OCT tended to underestimate central corneal thickness (CCT), but to overestimate the ACD in comparison to Scheimpflug photography (29). Secondary, the algorithms used to calculate RAL by two devices are different. CASIA2 takes the vertex of the lens anterior capsule as the surface center, and uses a three dimensional (3D) surface with a $4 \mathrm{~mm}$-diameter to fit and calculate the RAL (30). On the other hand, in this study, the algorithm to calculate RAL with Scheimpflug imaging averaged the measurements of three cross-sectional images from one eye to calculate the radius of the best fitting circle. The lens anterior surface with the diameter of about $6 \mathrm{~mm}$ in the pupil area was included. Previous studies using cadaveric eyes, which allowed observation of the whole anterior surface of the crystalline lens towards the equator, have shown that the anterior lens surface steepens toward the periphery, so the calculated RAL might decrease when the diameter of the anterior surface included in the fitting model was larger $(31,32)$. The abovementioned theories support and explain that the difference in RAL measurements between the two instruments is constant rather than disordered, and also indicate that the result from one instrument could be converted to that from another through linear regression equation.

The interpretation and application of the results of this study should take into account its limitations The correlation of the two measurement methods for the elders was not as good as that for the young subjects. Therefore, the conversion between RAL obtained with these two methods should be carried out with cautious in elders. In addition, we only evaluated the consistency between the two methods under mydriasis, and the results may not be directly extended to the data under non-mydriasis and accommodation condition.

\section{Conclusions}

In conclusion, our study found that both the Scheimpflug camera system with build-in caliper tool and the AS-OCT could measure the RAL successfully. The two measurement results are highly correlated and interchangeable through linear transformation. These fast and non-contact tools enable us to collect large-scale in vivo data of RAL to provide theoretical support for the study of the refractive and accommodation functions of the lens.

\section{Acknowledgments}

Funding: This study was supported by the National Natural Science Foundation of China $(81873675,81770905)$, and the Construction Project of High-Level Hospitals in Guangdong Province (303020102).

\section{Footnote}

Conflicts of Interest: The authors have no conflicts of interest to declare.

Ethical Statement: The authors are accountable for all aspects of the work in ensuring that questions related to the accuracy or integrity of any part of the work are appropriately investigated and resolved. All the procedures in this study were arranged strictly with the approval of the institutional review board of Zhongshan Ophthalmic Centre of Sun Yat-sen University (IRB-ZOC-SYSU). Written informed consents have been obtained from all participants.

Open Access Statement: This is an Open Access article distributed in accordance with the Creative Commons Attribution-NonCommercial-NoDerivs 4.0 International License (CC BY-NC-ND 4.0), which permits the noncommercial replication and distribution of the article with the strict proviso that no changes or edits are made and the original work is properly cited (including links to both the formal publication through the relevant DOI and the license). See: https://creativecommons.org/licenses/by-nc-nd/4.0/.

\section{References}

1. Donaldson PJ, Grey AC, Maceo Heilman B, et al. The 
physiological optics of the lens. Prog Retin Eye Res 2017;56:e1-24.

2. Satoh N, Shimizu K, Goto A, et al. Accommodative changes in human eye observed by Kitasato anterior segment optical coherence tomography. Jpn J Ophthalmol 2013;57:113-9.

3. Yuan Y, Shao Y, Tao A, et al. Ocular anterior segment biometry and high-order wavefront aberrations during accommodation. Invest Ophthalmol Vis Sci 2013;54:7028-37.

4. Croft MA, McDonald JP, Katz A, et al. Extralenticular and lenticular aspects of accommodation and presbyopia in human versus monkey eyes. Invest Ophthalmol Vis Sci 2013;54:5035-48.

5. Reilly MA. A quantitative geometric mechanics lens model: insights into the mechanisms of accommodation and presbyopia. Vision Res 2014;103:20-31.

6. Mateo T, Chang A, Mofid Y, et al. Axial ultrasound B-scans of the entire eye with a 20-MHz linear array: correction of crystalline lens phase aberration by applying Fermat's principle. IEEE Trans Med Imaging 2014;33:2149-66.

7. Lee YE, Joo CK. Assessment of Lens Center Using Optical Coherence Tomography, Magnetic Resonance Imaging, and Photographs of the Anterior Segment of the Eye. Invest Ophthalmol Vis Sci 2015;56:5512-8.

8. de Castro A, Benito A, Manzanera S, et al. ThreeDimensional Cataract Crystalline Lens Imaging With Swept-Source Optical Coherence Tomography. Invest Ophthalmol Vis Sci 2018;59:897-903.

9. Puk $\mathrm{O}$, de Angelis MH, Graw J. Lens density tracking in mice by Scheimpflug imaging. Mamm Genome 2013;24:295-302.

10. Lin H, Lin D, Liu Z, et al. A Novel Congenital Cataract Category System Based on Lens Opacity Locations and Relevant Anterior Segment Characteristics. Invest Ophthalmol Vis Sci 2016;57:6389-95.

11. Doyle L, Little JA, Saunders KJ. Repeatability of OCT lens thickness measures with age and accommodation. Optom Vis Sci 2013;90:1396-405.

12. Dunne MC, Davies LN, Wolffsohn JS. Accuracy of cornea and lens biometry using anterior segment optical coherence tomography. J Biomed Opt 2007;12:064023.

13. Fu J, Wang X, Li S, et al. Comparative study of anterior segment measurement with Pentacam and anterior segment optical coherence tomography. Can J Ophthalmol 2010;45:627-31.

14. González-Pérez J, Queiruga Piñeiro J, Sánchez García Á, et al. Comparison of Central Corneal Thickness Measured by Standard Ultrasound Pachymetry, Corneal Topography, Tono-Pachymetry and Anterior Segment Optical Coherence Tomography. Curr Eye Res 2018;43:866-72.

15. Savini G, Schiano-Lomoriello D, Hoffer KJ. Repeatability of automatic measurements by a new anterior segment optical coherence tomographer combined with Placido topography and agreement with 2 Scheimpflug cameras. J Cataract Refract Surg 2018;44:471-8.

16. Oatts JT, Keenan JD, Mannis T, et al. Multimodal Assessment of Corneal Thinning Using Optical Coherence Tomography, Scheimpflug Imaging, Pachymetry, and SlitLamp Examination. Cornea 2017;36:425-30.

17. British Standards Institute. Accuracy (Trueness and Precision) of Measurement Methods and Results: Basic Methods for the Determination of Repeatability and Reproducibility of a Standard Measurement Method. London: HMO, 1994.

18. Rosen AM, Denham DB, Fernandez V, et al. In vitro dimensions and curvatures of human lenses. Vision Res 2006;46:1002-9.

19. Rosales P, Dubbelman M, Marcos S, et al. Crystalline lens radii of curvature from Purkinje and Scheimpflug imaging. J Vis 2006;6:1057-67.

20. Koretz JE, Strenk SA, Strenk LM, et al. Scheimpflug and high-resolution magnetic resonance imaging of the anterior segment: a comparative study. J Opt Soc Am A Opt Image Sci Vis 2004;21:346-54.

21. Richdale K, Sinnott LT, Bullimore MA, et al. Quantification of age-related and per diopter accommodative changes of the lens and ciliary muscle in the emmetropic human eye. Invest Ophthalmol Vis Sci 2013;54:1095-105.

22. Ramasubramanian V, Glasser A. Prediction of accommodative optical response in prepresbyopic subjects using ultrasound biomicroscopy. J Cataract Refract Surg 2015;41:964-80.

23. Hermans EA, Pouwels PJ, Dubbelman M, et al. Constant volume of the human lens and decrease in surface area of the capsular bag during accommodation: an MRI and Scheimpflug study. Invest Ophthalmol Vis Sci 2009;50:281-9.

24. Schröder S, Mäurer S, Eppig T, et al. Comparison of Corneal Tomography: Repeatability, Precision, Misalignment, Mean Elevation, and Mean Pachymetry. Curr Eye Res 2018;43:709-16.

25. Xu Z, Peng $M$, Jiang J, et al. Reliability of Pentacam HR Thickness Maps of the Entire Cornea in Normal, PostLaser In Situ Keratomileusis, and Keratoconus Eyes. Am J 
Ophthalmol 2016;162:74-82.e1.

26. Chansangpetch S, Nguyen A, Mora M, et al. Agreement of Anterior Segment Parameters Obtained From SweptSource Fourier-Domain and Time-Domain Anterior Segment Optical Coherence Tomography. Invest Ophthalmol Vis Sci 2018;59:1554-61.

27. Shoji T, Kato N, Ishikawa $S$, et al. In vivo crystalline lens measurements with novel swept-source optical coherent tomography: an investigation on variability of measurement. BMJ Open Ophthalmol 2017;1:e000058.

28. Fu Y, Yu X, Savini G, et al. Assessment of Corneal Keratometric and Astigmatism Measurements Using Verion System and Other Instruments in Cataract Patient. Curr Eye Res 2018;43:1205-14.

29. Wang Q, Ding X, Savini G, et al. Anterior chamber depth measurements using Scheimpflug imaging and optical coherence tomography: repeatability, reproducibility, and agreement. J Cataract Refract Surg 2015;41:178-85.

30. Fukuda S, Ueno Y, Fujita A, et al. Comparison of anterior segment and lens biometric measurements in patients with cataract. Graefes Arch Clin Exp Ophthalmol 2020;258:137-46.

31. Manns F, Fernandez V, Zipper S, et al. Radius of curvature and asphericity of the anterior and posterior surface of human cadaver crystalline lenses. Exp Eye Res 2004;78:39-51.

32. Ortiz S, Siedlecki D, Grulkowski I, et al. Optical distortion correction in optical coherence tomography for quantitative ocular anterior segment by three-dimensional imaging. Opt Express 2010;18:2782-96.

Cite this article as: Liu Z, Ruan X, Wang W, Liu J, Meng Y, $\mathrm{Gu}$ X, Fu J, Luo L, Liu Y. Comparison of radius of anterior lens surface curvature measurements in vivo using the anterior segment optical coherence tomography and Scheimpflug imaging. Ann Transl Med 2020;8(5):177. doi: 10.21037/ atm.2020.01.100 